\title{
Critical role of TSLP-responsive mucosal dendritic cells in the induction of nasal antigen-specific lgA
} response

\author{
S Joo ${ }^{1,2}$, Y Fukuyama ${ }^{1}$, EJ Park ${ }^{1,3}$, Y Yuki ${ }^{1}$, Y Kurashima ${ }^{1,2}$, R Ouchida ${ }^{1}$, SF Ziegler ${ }^{4}$ and H Kiyono ${ }^{1,2,5,6}$
}

Thymic stromal lymphopoietin (TSLP) is an interleukin-7 (IL-7)-like cytokine involved in T helper 2 type immune responses. The primary target of TSLP is myeloid dendritic cells (DCs), however, little is known about the mechanism by which TSLP elicits respiratory IgA immune responses upon mucosal immunization. Here, we found that the levels of TSLP and TSLPR were upregulated in the mucosal DCs of mice nasally immunized with pneumococcal surface protein $A$ (PspA) plus cholera toxin (CT) compared with those immunized with PspA alone. PspA-specific IgA responses, but not IgG Ab responses were significantly reduced in both serum and mucosal secretions of TSLPR knockout mice compared with wild-type mice after nasal immunization with PspA plus CT. Furthermore, CD11c ${ }^{+}$mucosal DCs isolated from TSLPR knockout mice nasally immunized with PspA plus CT were less activated and exhibited markedly reduced expression of IgA-enhancing cytokines (e.g., APRIL, BAFF, and IL-6) compared with those from equivalently immunized wild-type mice. Finally, exogenous TSLP promoted production of IgAs in an in vitro DC-B cell co-culture system as exhibited by enhanced IL-6 production. These results suggest that TSLP-TSLPR signaling is pivotal in the induction of nasal respiratory immunity against pathogenic pneumococcal infection.

\section{INTRODUCTION}

The current demand for new mucosal vaccine and adjuvant treatments to prevent life-threatening microbial infections is great. Mucosal vaccines, especially nasally administered ones, have advantages over conventional systemic vaccines because mucosal vaccines can induce antigen (Ag)-specific protective immunity in both the mucosal and systemic immune systems. ${ }^{1,2}$ Thus, mucosal vaccines have been considered to be ideal for prevention and protection from pathogens invading through mucosal sites. The adaptive humoral immune defense at mucosal surfaces is predominantly provided by secretory IgA (SIgA) antibodies (Abs). ${ }^{3}$ Mucosal SIgA Abs act as a front-line barrier in protection against pathogens, including Streptococcus pneumoniae by inhibiting attachment and invasion of mucosal surfaces. ${ }^{4,5}$ Therefore, a strategy to elicit Ag-specific SIgA responses will be crucial for designing efficacious mucosal vaccines. However, the molecular pathways underlying the induction and maintenance of $\mathrm{Ag}$-specific IgA responses at mucosal sites, especially airway tracts after mucosal immunization are still largely unknown.

Thymic stromal lymphopoietin (TSLP) is an interleukin-7 (IL-7)-like cytokine and is predominantly expressed by epithelial cells of barrier surfaces (skin, airway, and intestine), as well as stromal cells, mast cells, and dendritic cells (DCs). ${ }^{6,7}$ TSLP acts by binding to a heterodimeric receptor complex consisting of TSLP receptor (TSLPR) and IL-7 receptor $\alpha$ chain $(\mathrm{IL}-7 \mathrm{R} \alpha)^{6,8}{ }^{6}$ TSLPR is expressed by several immune cell types including DCs, mast cells, eosinophils, and T cells. ${ }^{9}$ Both human and mouse studies have shown that TSLP is involved in initiating various immune responses by contributing to the activation and maturation of DCs, B cells, and T cells. ${ }^{6,10}$ TSLP has important roles in conditioning DCs to drive T helper 2 (Th2) differentiation and the production of TSLP is increased in immunopathologies associated with dysregulated Th2-type

${ }^{1}$ Division of Mucosal Immunology, Department of Microbiology and Immunology, The Institute of Medical Science, The University of Tokyo, Tokyo, Japan. ${ }^{2}$ Graduate School of Medicine and Faculty of Medicine, The University of Tokyo, Tokyo, Japan. ${ }^{3}$ Department of Molecular Pathobiology and Cell Adhesion Biology, Mie University Graduate School of Medicine, Mie, Japan. ${ }^{4}$ Immunology Program, Benaroya Research Institute, Seattle, Washington, USA. ${ }^{5}$ International Research and Development Center for Mucosal Vaccines, The Institute of Medical Science, The University of Tokyo, Tokyo, Japan and ${ }^{6}$ Department of Immunology, Graduate School of Medicine, Chiba University, Chiba, Japan. Correspondence: H Kiyono (kiyono@ims.u-tokyo.ac.jp) 
cytokine production. ${ }^{11,12,13}$ Although, most studies of TSLP have focused on its role in allergic inflammation in skin and airways, ${ }^{11,12,14}$ TSLP has also been reported to have important roles in $\mathrm{Ab}$ class-switching recombination in humans. ${ }^{15,16}$ Other studies from two independent groups suggest that exogenous TSLP may be a promising adjuvant for cytotoxic T-cell expansion ${ }^{17}$ and for nasal immunization. ${ }^{18}$ However, whether endogenous TSLP regulates Ab production in mucosal immunization remains unclear.

To address this issue here, we used a pneumococcal surface protein A (PspA) nasal immunization model. ${ }^{19}$ Pneumococcal infection by $S$. pneumoniae is the major cause of virulent respiratory infectious disease in humans, which causes otitis media, pneumonia, and meningitis. ${ }^{20} \mathrm{PspA}$ is a cholinebinding surface protein of $S$. pneumoniae; it is commonly expressed by all capsular strains of $S$. pneumoniae and is highly cross-reactive between mice and humans. ${ }^{21}$ Nasal administration of PspA plus adjuvant (e.g., cholera toxin (CT) or CT B subunit) preferentially induces PspA-specific $\mathrm{Ab}$ responses in mice and provides protective immunity against pneumococcus, and thus is a potent nasal vaccine $\mathrm{Ag}$ for pneumococcal vaccines. ${ }^{19,22,23}$

Here we revealed a new immunological role for TSLP in vivo in nasal PspA-specific IgA. We showed that TSLP expression is highly upregulated in the mucosal tissues of mice nasally immunized with PspA plus CT (which is a Th2-type adjuvant), suggesting that the TSLP-TSLPR interaction acts as a cue for mediating PspA-specific mucosal immunity. Our results indicate that the TSLP-TSLPR pathway is critical for induction of Ag-specific SIgA responses upon intranasal immunization with PspA plus CT. In addition, by using an in vitro DC-B cell co-culture system we demonstrated that TSLP treatment enhances IgA production in an IL-6-dependent manner. These findings increase our understanding of the role of TSLP signaling in humoral immunity upon mucosal immunization and will therefore facilitate the development of more effective and beneficial mucosal vaccines and adjuvants.

\section{RESULTS}

\section{Upregulation of TSLP in the respiratory tissues of mice given nasal PspA plus CT}

We initially examined whether nasal PspA with adjuvant enhanced TSLP expression in respiratory tissues. We isolated total RNA from airway tissues, including nasal passage (NP) and lung, of nasally immunized mice and assessed TSLP expression by real-time-quantitative (RT-qPCR). TSLP messenger RNA (mRNA) levels in NP and lung tissue were significantly enhanced in mice nasally immunized with PspA plus CT compared with naive (administered phosphatebuffered saline (PBS) only) mice or mice nasally immunized with PspA only, CT only, or PspA plus CpG oligonucleotides (CpG ODN; adjuvant that induces Th1 cytokine production ${ }^{24}$ ) (Figure 1a). Enzyme-linked immunosorbent assay (ELISA) analysis of the broncho-alveolar fluid from these mice detected significantly higher TSLP protein levels in those nasally immunized with PspA plus CT compared with those in the control groups (Figure 1b). These data indicate that TSLP expression is induced in the respiratory tissues of mice by nasal immunization with PspA plus CT, which is a representative Th2-type adjuvant; this result suggests the possible involvement of TSLP in Ab production by nasal immunization.

\section{Enhanced expression of TSLP and TSLPR in mucosal CD11c $^{+}$DCs after nasal immunization with PspA plus CT} To identify which cells produce TSLP in response to nasal immunization, we performed immunofluorescence staining of lung tissue and nasopharynx-associated lymphoid tissue (NALT) of nasally immunized mice. In both these tissue types, the level of TSLP was higher in mice given nasal PspA plus CT than in those given PspA only (Supplementary Figure S1a, b online). Furthermore, analysis of the co-localization of staining for TSLP and CD11c showed that TSLP expression in CD11c ${ }^{+}$DCs and epithelial cells of lung and NALT tissue was significantly greater in PspA plus CT nasally immunized mice than in the PspA only group (Supplementary Figure S1a,b). To confirm the TSLP expression in CD11c ${ }^{+}$DCs, we conducted RT-qPCR analysis to measure TSLP mRNA levels in $\mathrm{CD} 11 \mathrm{c}^{+} \mathrm{DCs}$ isolated from mucosa-associated tissues (NALT, $\mathrm{NP}$, and lung) of nasally immunized mice; in all three tissues, TSLP mRNA levels were significantly and highly upregulated in response to PspA plus CT nasal immunization compared with PspA only (Figure 2a). In contrast, TSLP mRNA expression in $\mathrm{CD}_{11 \mathrm{c}^{+}}$DCs from peripheral lymphoid tissue (spleen, cervical lymph node) was not significantly elevated by nasal PspA plus CT (Figure 2a). We next examined TSLPR expression in $\mathrm{CD} 11 \mathrm{~b}^{+} \mathrm{CD} 11 \mathrm{c}^{+}$DCs, a TSLP-responsive DC subset of immune cells, ${ }^{25,26}$ from the relevant tissues of nasally immunized mice. Flow cytometric analysis showed that after nasal immunization with PspA plus CT, TSLPR expression significantly increased in $\mathrm{CD} 11 \mathrm{~b}^{+} \mathrm{CD} 11 \mathrm{c}^{+} \mathrm{DCs}$ from NP and lung tissue but not in those from spleen tissue (Figure 2b). Taken together, these data suggest that TSLP produced by mucosal epithelial cells and DCs in response to nasally administered PspA plus CT may trigger the TSLPR-mediated DC activation in nasal mucosal tissue.

\section{PspA-specific IgA Ab responses were abrogated in TSLPR-KO mice after nasal immunization with PspA plus CT}

We next used TSLPR knockout (KO) mice to investigate whether the abrogation of TSLP-TSLPR signaling affects Agspecific $\mathrm{Ab}$ responses upon nasal immunization. We nasally immunized wild-type (WT) or TSLPR-KO mice with PspA plus CT a total of three times at 1-week intervals and harvested samples 1 week after the last immunization. TSLPR deficiency had no significant effect on anti-PspA IgG Ab responses in serum or nasal washes (Figure 3a); in addition, there was no significant change in the level of PspA-specific serum IgG1 and no detectable IgG2 responses in either immunized WT or TSLPR-KO mice (Supplementary Figure S2). In contrast, TSLPR deficiency significantly reduced PspA-specific IgA responses in both serum and mucosal excretions (Figure 3a). These findings were confirmed at the cellular level, in that the 
a

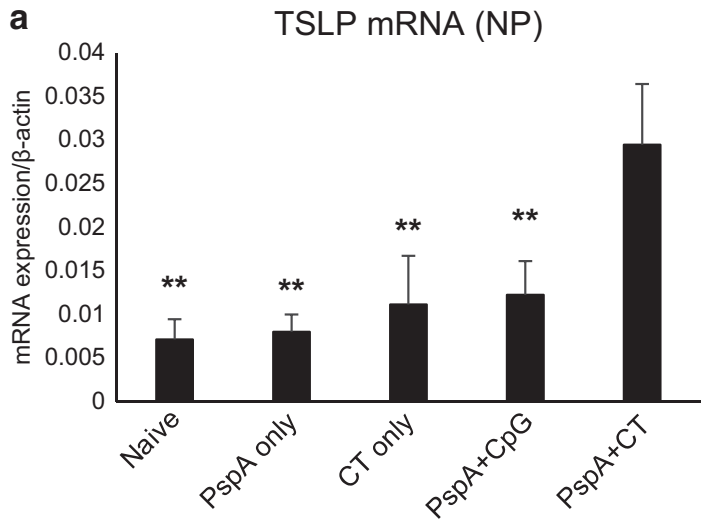

b

b $100 \quad$ TSLP protein (BALF)

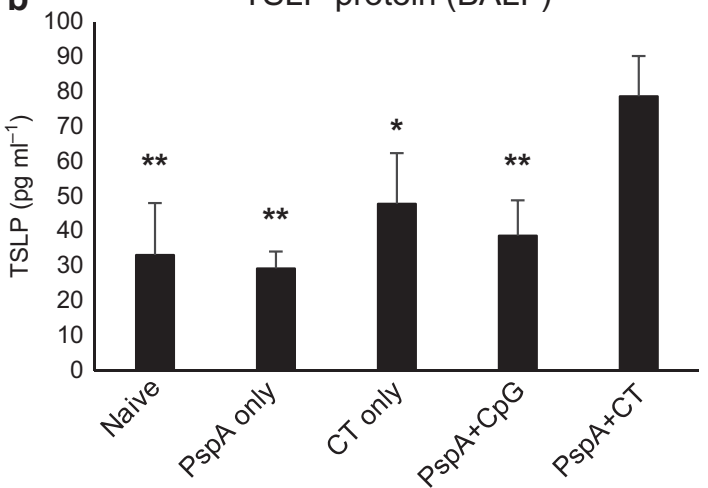

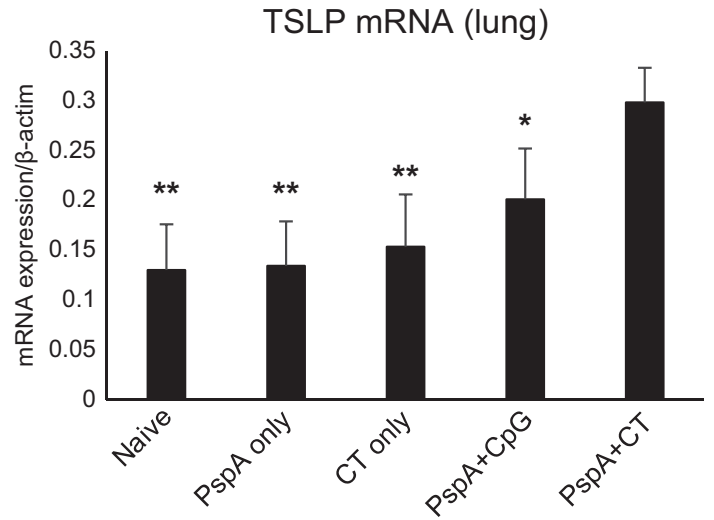

Figure 1 TSLP expression in nasally immunized mice. Each mouse group was nasally immunized weekly for 3 consecutive weeks with PBS (naive), $5 \mu \mathrm{g}$ PspA only, $1 \mu \mathrm{g} \mathrm{CT}$ only, $5 \mu \mathrm{g}$ PspA plus $10 \mu \mathrm{g}$ CpG ODN, or $5 \mu \mathrm{g}$ PspA plus $1 \mu \mathrm{g} \mathrm{CT}$. All the samples were harvested at 1 week after the last immunization. (a) RT-qPCR analysis of TSLP mRNA expression in NP and lung tissues. The mRNA expression level was normalized to that of $\beta$ actin (b) Detection of TSLP protein level in BALF by ELISA. Values are means \pm s.d. ( $n=4$ per group). ${ }^{\star} P<0.05,{ }^{\star *} P<0.01$ vs. mice nasally immunized with PspA plus CT. Data are representative of two independent experiments. BALF, broncho-alveolar fluid; CT, cholera toxin; ELISA, enzyme-linked immunosorbent assay; mRNA, messenger RNA; NP, nasal passages; PBS, phosphate-buffered saline; PspA, pneumococcal surface protein A; RT-qPCR, real-time-quantitative PCR; TSLP, thymic stromal lymphopoietin.

numbers of PspA-specific IgA Ab-forming cells significantly decreased in spleen and NP of TSLPR-KO mice given PspA plus CT when compared with identically immunized WT mice (Figure 3b). Despite the lack of PspA-specific IgA responses in TSLPR-KO mice immunized with PspA plus CT, the total immunoglobulin levels in serum and mucosal secretions were comparable between WT and TSLPR-KO mice in the steady state (Supplementary Figure S3), which was consistent with a previous finding. ${ }^{27}$ These results clearly demonstrate that TSLPTSLPR signaling is essential for the induction of Ag-specific IgA responses after nasal immunization with PspA plus CT.

\section{TSLPR-KO mice given nasal PspA plus CT showed high} colonization of $S$. pneumoniae after nasal challenge

To explore the functional role of TSLP-TSLPR-mediated induction of PspA-specific mucosal SIgA Abs in the prevention of bacterial colonization in respiratory tissue, WT, and TSLPRKO mice were nasally immunized with PspA plus CT or PspA only and then nasally challenged with $S$. pneumoniae Xen10 strain 1 week after the last immunization. The numbers of colony-forming units (CFUs) in TSLPR-KO mice were similar to those in WT mice challenged with Xen10 strain when they were given nasal PspA only (Figure $\mathbf{4 a}, \mathbf{b}$ ). Consistent with previous studies, ${ }^{5,28}$ WT mice immunized with PspA plus CT showed essentially no bacteria in NP or lung (Figure 4a,b). In contrast, large numbers of bacterial CFUs were detected in NP and lung of TSLPR-KO mice immunized with PspA plus CT (Figure 4a, b). These results clearly demonstrate that TSLPRKO mice given nasal PspA plus CT were impaired in their ability to suppress bacterial colonization despite the presence of comparably high levels of PspA-specific IgG Abs; we consider that this effect is likely due to a lack of Ag-specific SIgA responses.

\section{Characterization of the IgA-enhancing abilities of CD11c ${ }^{+}$ DCs from PspA plus CT nasally immunized mice}

We next addressed the mechanism by which TSLP signaling is involved in Ag-specific IgA responses. To do this, we focused on the function of $\mathrm{CD} 11 \mathrm{c}^{+}$DCs because they were responsive to nasal immunization with PspA plus CT (Figure 2a,b), they had the highest TSLPR expression in spleen and lung tissue among immune cells analyzed (Supplementary Figure S4), and they are known to be the primary target of TSLP by several lines of previous evidence. ${ }^{8,9,25,29}$ To assess whether TSLPR signaling 
a

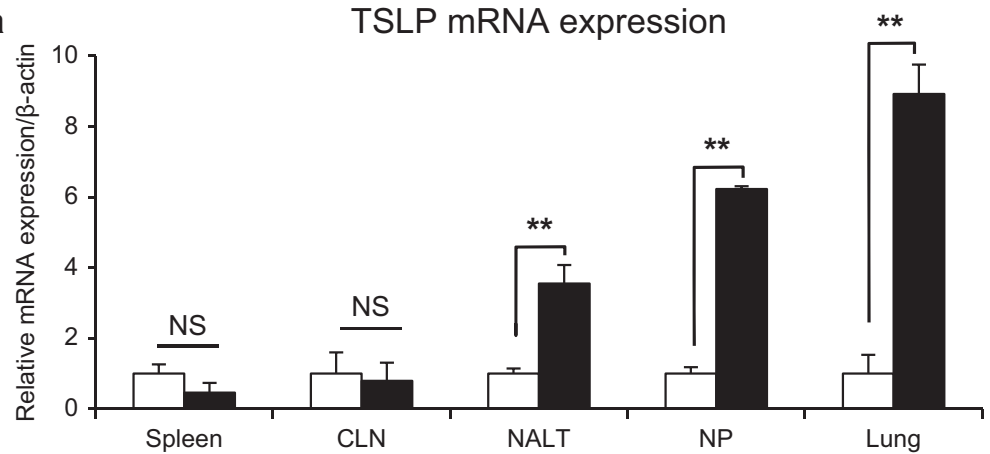

口PspA only DCs

PspA+CT DCs

b

CD11 ${ }^{+}$CD11 $\mathrm{c}^{+}$gated
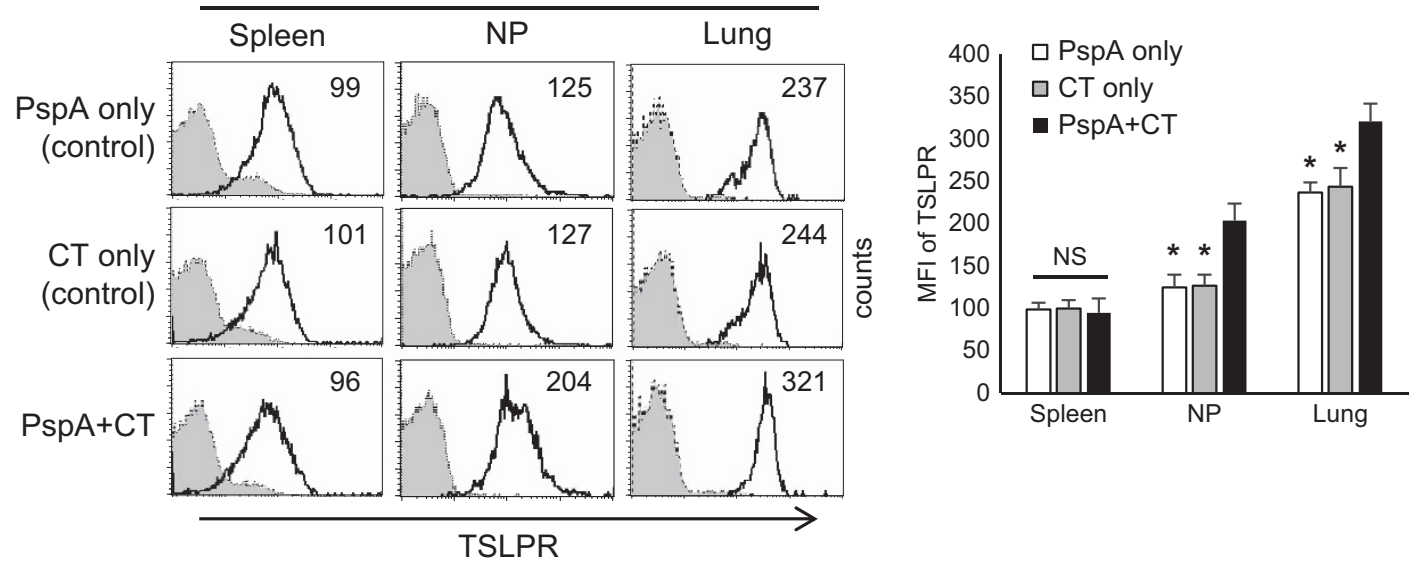

TSLPR

Figure 2 TSLP and TSLPR expression in DCs of nasally immunized mice. Mice were nasally immunized weekly for 3 consecutive weeks with PspA only, CT only, or PspA plus CT. All the samples were harvested at 1 week after the last immunization. (a) RT-qPCR analysis of TSLP mRNA expression in CD11c ${ }^{+}$DCs from spleen, CLN, NALT, NP, and lung tissue of nasally immunized mice. CD11c ${ }^{+}$DCs were isolated by MACS and pooled from three mice per group. The mRNA expression level was normalized to that of $\beta$ actin, and data are expressed relative to the value in PspA only DCs. Values represent the mean \pm s.e.m. of values from three independent experiments. ${ }^{*} P<0.01$ vs. PspA only DCs. (b) Flow cytometric analysis of TSLPR expression in

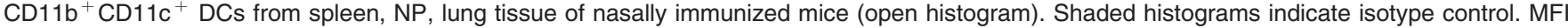
indicating TSLPR expression is shown. Values are means \pm s.e.m. $(n=3$ per group). Data are representative of three independent experiments. ${ }^{\star} P<0.05$ vs. mice nasally immunized with PspA plus CT. CLN, cervical lymph node; CT, cholera toxin; DC, dendritic cells; MACS, magnetic-activated cell sorting; MFI, mean fluorescence intensity; mRNA, messenger RNA; NP, nasal passages; NS, not significant; PspA, pneumococcal surface protein A; RTqPCR, real-time-quantitative PCR; TSLP, thymic stromal lymphopoietin.

had any effect on the activation status of the CD11c ${ }^{+}$DC population in various tissues after nasal immunization, we determined the protein expression of MHC II molecules and DC costimulatory molecules (CD40 and CD86). Flow cytometric analysis showed that DCs isolated from mucosaassociated tissues (NALT, NP, and lung) but not from spleen of TSLPR-KO mice immunized with PspA plus CT exhibited lower expression of the MHC class II I-A $\mathrm{A}^{\mathrm{d}}$ antigen, CD40, and CD86 than did those from equivalently immunized WT mice (Figure 5a). However, in the steady state, the absence of TSLPTSLPR signaling had no effect on the phenotype of CD11c ${ }^{+}$ DCs (Supplementary Figure S5). We next investigated the mRNA expression of IgA-enhancing factors in CD11 ${ }^{+}$DCs isolated from PspA plus CT-immunized mice. Expression of mRNAs encoding retinaldehyde dehydrogenase type 2 (Aldh1a2), which mediates retinoic acid production, APRIL, BAFF, iNOS, TGF- $\beta$, and IL- 6 and -10 was lower in DCs from mucosa-associated tissues of TSLPR-KO mice immunized with PspA plus CT than in those from the equivalently immunized
WT mice (Figure 5b); in contrast, expression of the above mRNAs in DCs from spleen showed no significant difference between immunized WT and TSLPR-KO mice (Figure 5b). These data indicate that, in the absence of TSLP-TSLPR signaling, CD11 $\mathrm{c}^{+}$DCs in mucosa-associated tissues, but not those in spleen, were less activated and lacked IgA-inducing abilities compared with those from WT mice after nasal immunization with PspA plus CT.

\section{TSLP promotes IgA production by enhancing IL- 6 production in DCs}

To clarify the role of TSLP in the induction of IgA Abs, we cultured WT $\mathrm{IgD}^{+}$naive B cells alone or together with WT splenic or nasal CD11c ${ }^{+}$DCs; the cells were cultured in the presence or absence of mouse recombinant TSLP (rTSLP) and anti-CD40 monoclonal $\mathrm{Ab}$, which was added as a substitute for T-cell help to exclude the influence of T-cell-derived cytokines. ${ }^{30}$ After 7 days of culture, rTSLP treatment significantly augmented IgA levels in the culture supernatants in both a 

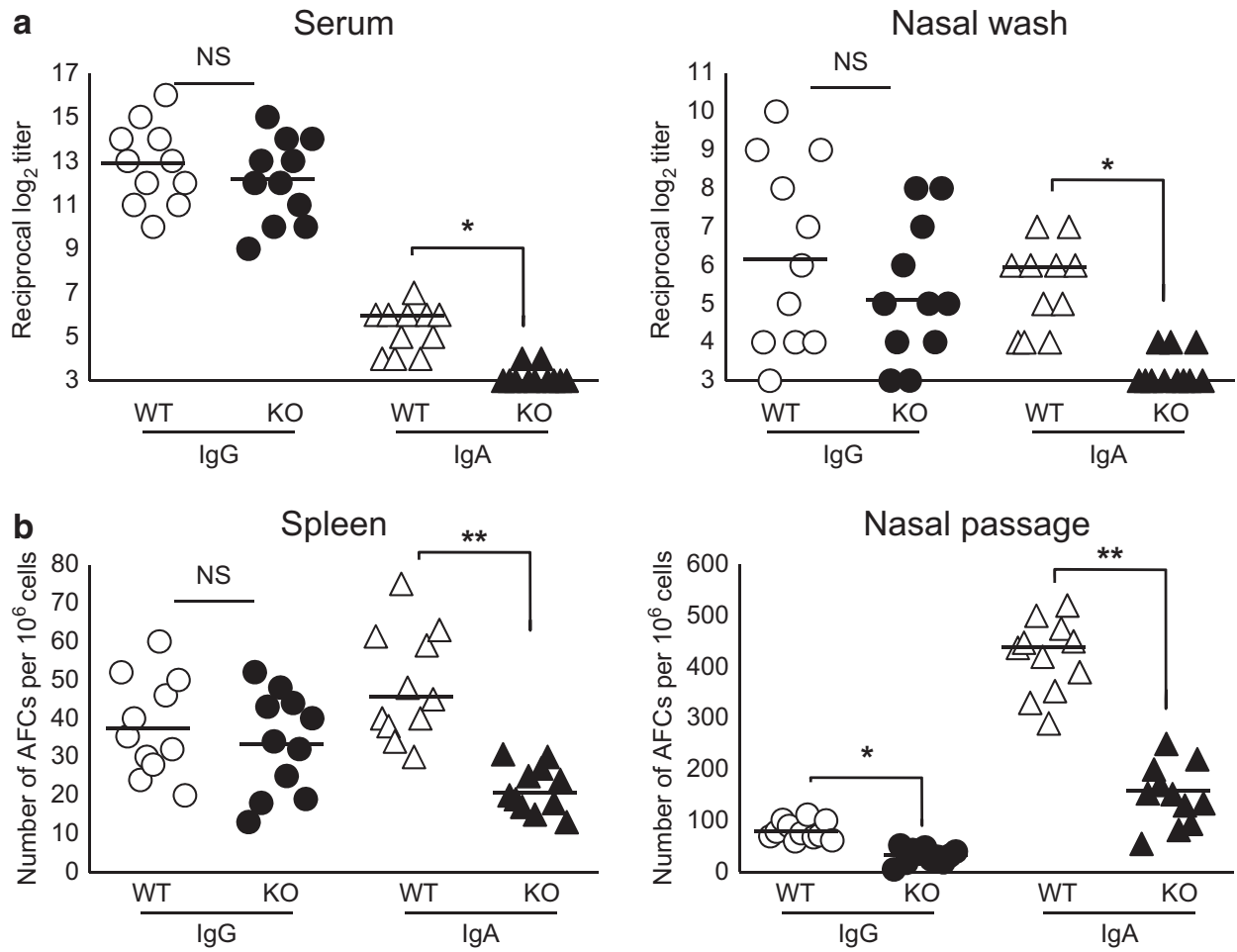

Figure 3 Reduced PspA-specific IgA responses in TSLPR-KO mice compared with WT mice after nasal PspA plus CT immunization. WT and TSLPRKO mice were nasally immunized with PspA plus CT weekly for 3 consecutive weeks and all the samples were harvested at 1 week after the last immunization. (a) The levels of anti-PspA IgG and IgA in serum and NW samples were determined by PspA-specific ELISA. (b) The numbers of anti-PspA IgG and IgA AFCs from spleen and NP were determined by PspA-specific ELISPOT assay. Graphs show data from individual mice, and bars indicate median. ${ }^{\star} P<0.05,{ }^{\star \star} P<0.01$. AFCs, Ab-forming cells; CT, cholera toxin; ELISA, enzyme-linked immunosorbent assay; ELISPOT, enzyme-linked immunospot; KO, knockout; NP, nasal passages; NS, not significant; NW, nasal washes; PspA, pneumococcal surface protein A; TSLP, thymic stromal lymphopoietin; WT, wild type.

CD40-dependent and -independent manner (Figure 6). The ability of DCs to promote IgA production after rTSLP treatment was more striking when the DCs were isolated from NP (NP-DCs) than when they were isolated from spleen (SP-DCs) (Figure 6). WT IgD ${ }^{+}$B cells cultured with rTSLP and anti-CD40 monoclonal $\mathrm{Ab}$ were capable of producing moderate but smaller amounts of IgA than when the same cells were cultured together with DCs (Figure 6). These results suggest that TSLP preferentially acts on the receptor-positive $\mathrm{CD} 11 \mathrm{c}^{+} \mathrm{DCs}$, rather than $\mathrm{B}$ cells, to augment IgA production in the DC-B cell co-culture system.

Next, to determine whether TSLP-stimulated DCs are associated with production of IgA-inducible cytokines, we measured the expression levels of various cytokines in the DC 2.4 cell line (derived from mouse bone marrow DCs) after stimulation with rTSLP for $48 \mathrm{~h}$. We first confirmed that the DC 2.4 cell line has a functional TSLP receptor complex by using flow cytometry to show that rTSLP stimulated the protein expression of both members of the heterodimeric receptor complex, TSLPR, and IL-7R $\alpha$ on DC 2.4 cells (Supplementary Figure S6a). RT-qPCR analysis detected a significant increase in the IL-6 mRNA level and a slight but significant increase in the BAFF mRNA level in TSLP-stimulated DC 2.4 cells when compared with non-treated cells; there was no significant difference in the levels of other cytokine mRNAs
(Supplementary Figure S6b). We therefore investigated whether freshly isolated and purified DCs produce IL-6. Significantly higher levels of IL- 6 were secreted by NP-DCs than by SP-DCs, and production from both types of DC was significantly decreased by anti-TSLP treatment and significantly increased by rTSLP stimulation (Figure 7a).

These observations led us to test the effect of IL- 6 on the IgA-inducing ability of TSLP. IL-6 production was significantly enhanced by rTSLP treatment in SP-DC or NP-DC plus B-cell co-cultures but not in monocultures of B cells (Figure 7b). To examine whether IL-6 is responsible for the TSLP-induced IgA production described in Figure 6, blocking Ab specific for IL-6 was added to the DC-B cell co-culture system. Induction of IgA production by rTSLP was attenuated by the neutralization of IL-6 (Figure 7c). Taken together, these results suggest that IL- 6 induced by TSLP-stimulated DCs has a major role in enhancing IgA production from DC-B cell co-cultures.

\section{DISCUSSION}

In the present study, we demonstrated the critical role of the TSLP-TSLPR pathway in the induction of Ag-specific SIgA responses through intranasal immunization. Earlier studies suggested that TSLP expression by epithelial cells might contribute to $\mathrm{Ab}$ isotype class switching in B cells in humans, 

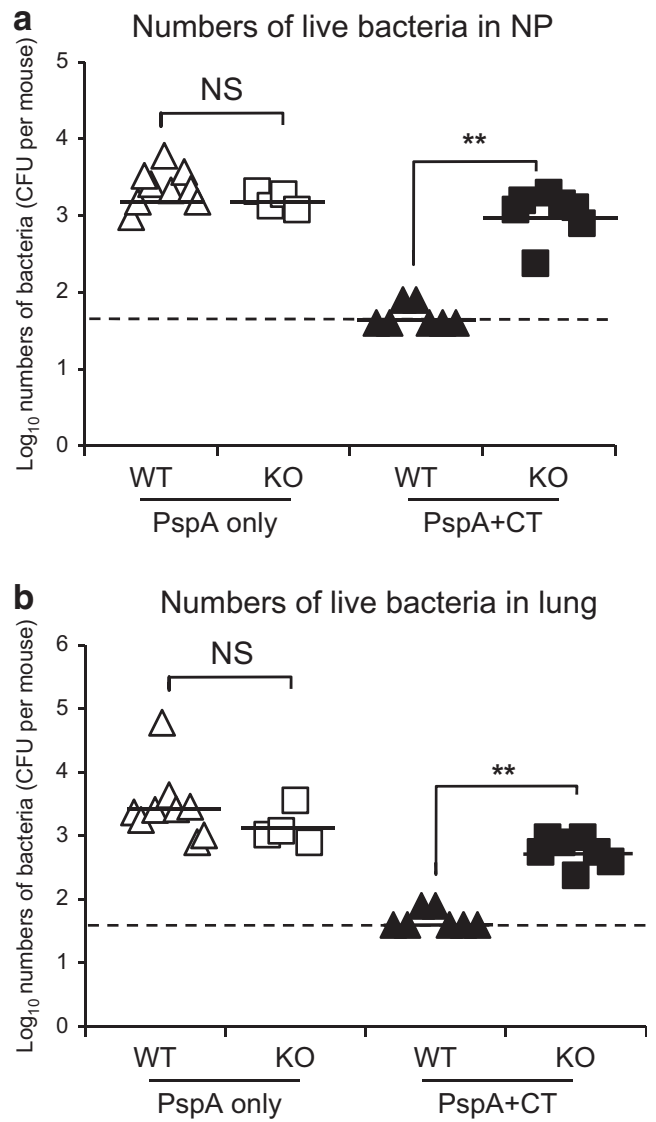

Figure 4 TSLPR-KO mice given nasal PspA plus CT impaired clearance of $S$. pneumoniae colonization. One week after the last immunization, both WT and TSLPR-KO mice were nasally challenged with $5 \times 10^{4} \mathrm{CFU}$ of $S$. pneumoniae strain Xen10. The numbers of bacterial colonies in (a) NP and (b) lung samples at 3 days after challenge. Data were combined from multiple experiments. Each solid line represents the median $\log _{10} \mathrm{CFU}$ per mouse; the dashed line represents no CFU detected. ${ }^{*} P<0.01 \mathrm{vs.} \mathrm{PspA}$ plus CT nasally immunized WT mice. CFU, colony-forming units; CT, cholera toxin; KO, knockout; NP, nasal passages; NS, not significant PspA, pneumococcal surface protein A; TSLP, thymic stromal lymphopoietin; WT, wild type.

by stimulating DCs to produce more APRIL ${ }^{15}$ or BAFF. ${ }^{16}$ Here, we found that TSLP mRNA and protein expression levels were significantly increased in respiratory tissues of mice nasally immunized with PspA plus CT compared with those immunized with PspA alone. To demonstrate whether the TSLPTSLPR interaction mediates humoral immune responses upon mucosal immunization, we examined Ag-specific Ab production in WT and TSLPR-KO mice after nasal immunization with PspA plus CT; use of CT as an adjuvant elicits Ag-specific Th2type responses and promotes the generation of Ag-specific IgAproducing $\mathrm{B}$ cells. ${ }^{22} \mathrm{Ag}$-specific IgA in both serum and mucosal secretions was significantly reduced in TSLPR-KO mice nasally immunized with PspA plus CT compared with that in equivalently immunized WT mice, whereas Ag-specific IgG responses were not affected (Figure 3a,b). TSLPR deficiency resulted in diminished bacterial clearance after bacterial challenge with $S$. pneumoniae (Figure $4 \mathbf{a}, \mathbf{b}$ ). These results indicate that TSLP-TSLPR interaction is required for the induction of Ag-specific IgA responses and protection of the respiratory tract against bacterial colonization.

Among diverse cells responding to TSLP, myeloid DCs are the best characterized. ${ }^{9,25,26,29}$ Here, CD11c ${ }^{+}$DCs showed higher expression of TSLPR than other immune cells $\left(\mathrm{CD} 3 \mathrm{e}^{+}\right.$ $\mathrm{T}$ cells and $\mathrm{CD} 19^{+}$B cells; Supplementary Figure S4). Previous studies showed that TSLP directly activates DCs by upregulating costimulatory molecules and "educating" DCs to promote Th2 differentiation both in mouse and human, ${ }^{13,26,29}$ and specific subsets of DCs, such as CD11b ${ }^{+}$DCs in mouse skin $^{25}$ and CD1c ${ }^{+}$DCs in human nasal mucosa, ${ }^{29}$ upregulate CCR7 and initiate Th2 differentiation in the draining lymph node in response to TSLP. Generally, DCs have a pivotal role in initiating the adaptive immune responses, in that they can recognize pathogens by innate receptors and activate naive $\mathrm{T}$ cells. ${ }^{31}$ However, DCs have also been implicated in class switching to IgA in naive B cells both in T-cell dependent and independent pathways. ${ }^{3}$ Mucosal DCs such as Peyer's patches DCs and lung DCs have more potent capability in driving IgA production than non-mucosal DCs, in that they produce higher levels of IgA-inducible cytokines such as APRIL, BAFF, IL-6, TGF- $\beta$, iNOS and express RALDH, which is converted into retinoic acid. $^{30,31}$

We observed that mucosal DCs produce and respond to TSLP after nasal immunization with PspA plus CT. The expression levels of TSLPR in $\mathrm{CD} 3 \mathrm{e}^{+} \mathrm{T}$ cells and $\mathrm{CD} 19^{+} \mathrm{B}$ cells from mucosal tissues, but not from spleen, were also upregulated upon immunization with PspA plus CT (Supplementary Figure S7a,b), although TSLPR expression in both cell types was lower (mean fluorescence intensity value, $\leqslant 20$ ) than that in $\mathrm{CD} 11 \mathrm{c}^{+} \mathrm{DCs}$. We therefore propose that TSLP exerts its action preferentially through DCs rather than other immune cells. Recent findings suggest that DCs produce TSLP in response to bacterial or allergen stimulation, which results both physiological and pathological immune regulation. ${ }^{7,32}$

From the above findings, we hypothesize that TSLP produced by mucosal DCs allows the direct activation of the adaptive immune response in an autocrine manner, which assists epithelial cell-derived TSLP to promote Ag-specific IgA humoral immune responses. Consistent with this hypothesis, we observed that in the absence of TSLP signaling, the activation status and expression levels of IgA-inducible cytokines were diminished to a greater extent in mucosal DCs than non-mucosal DCs from mice immunized with PspA plus CT (Figure 5a,b). Therefore, DCs from mucosal tissues are more influenced by the absence of TSLP-TSLPR signaling than are non-mucosal DCs; however, the key subsets of TSLP-responsive mucosal DCs involved in the response to nasal PspA plus CT remain to be fully characterized. We found that macrophages have comparable or lower TSLPR expression, and $\mathrm{CD} 8 \alpha^{+}$and $\mathrm{CD} 103^{+}$DCs have slightly higher TSLPR expression, compared with that in CD11b ${ }^{+}$DCs in various tissues (Supplementary Figure S4). Whether these specific $\mathrm{CD} 103^{+} \mathrm{DC}$ subsets in nasal mucosal tissues respond to TSLP induced by nasal immunization will be investigated in the near future. 
a
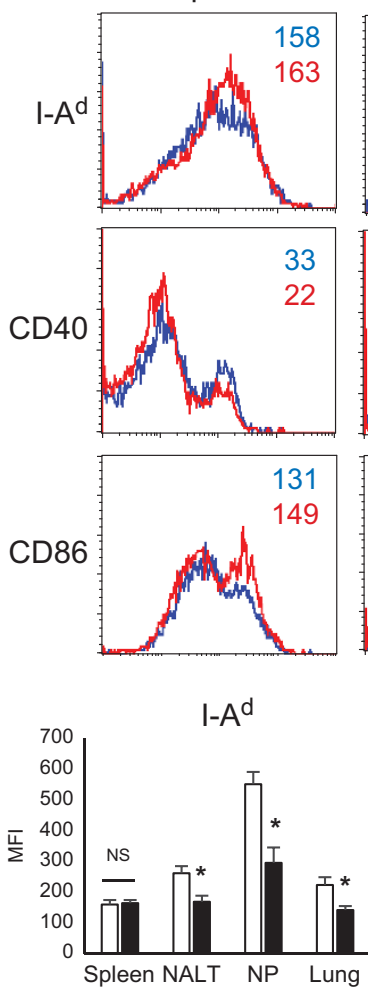

NALT
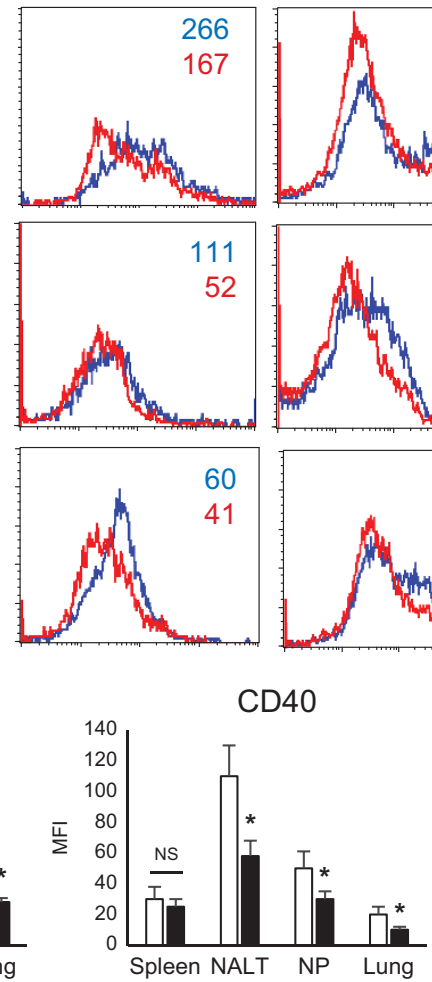

NP
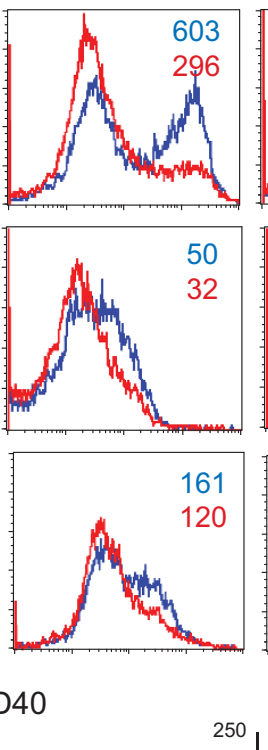
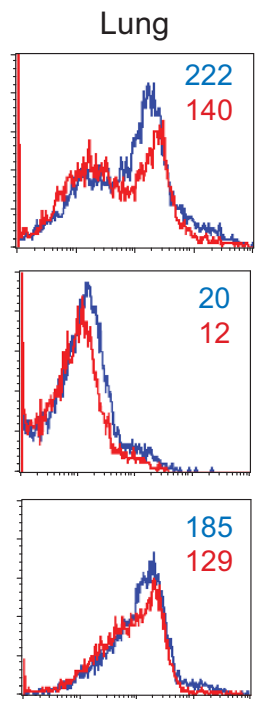
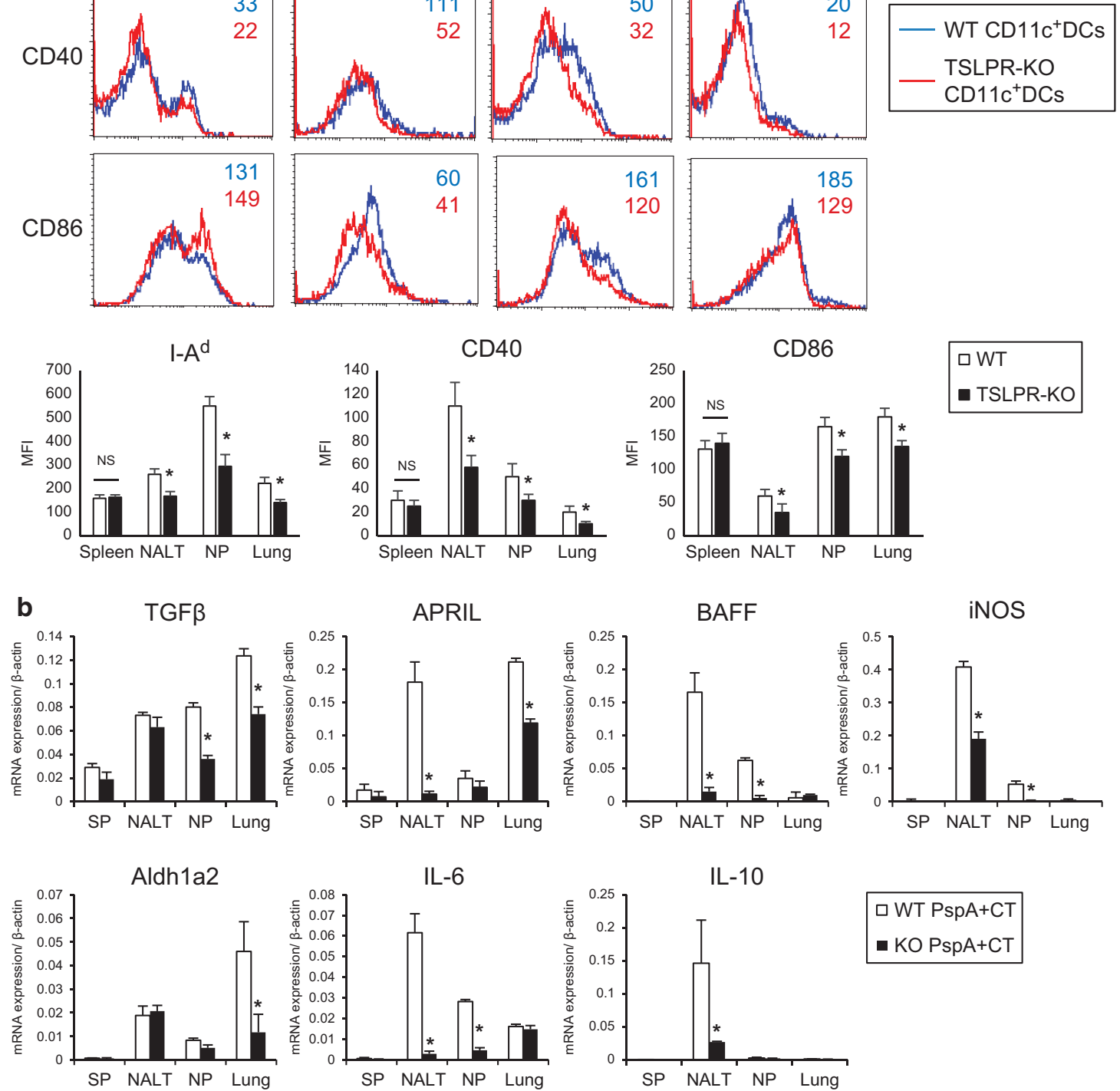

Figure 5 Comparison of phenotypes of CD11c ${ }^{+}$DCs from PspA plus CT nasally immunized mice. WT and TSLPR-KO mice were each nasally immunized with PspA plus CT weekly for 3 consecutive weeks, and tissue samples were harvested at 1 week after the last immunization. (a) Surface marker staining of CD11 ${ }^{+}$DCs in the indicated tissues from nasally immunized WT or TSLPR-KO mice. Numbers show MFI levels of indicated markers. Data are representative of three independent experiments ( $n=2$ or 3 per group). MFI indicating I- $\mathrm{A}^{\mathrm{d}}, \mathrm{CD} 40, \mathrm{CD} 86$ expression is shown. Values are means \pm s.d. ( $n=6$ or 7 per group). ${ }^{*} P<0.05$ vs. PspA plus CT nasally immunized WT mice. (b) mRNA levels expressed relative to $\beta$ actin mRNA for the indicated genes in the indicated tissues as determined for CD11c ${ }^{+}$DCs from nasally immunized WT or TSLPR-KO mice. CD11c ${ }^{+}$DCs were isolated by MACS and pooled from three mice per group. Values represent the means \pm s.e.m. of values from three independent experiments. ${ }^{*} P<0.05 \mathrm{vs}$. DCs from PspA plus CT nasally immunized WT mice. CT, cholera toxin; DC, dendritic cells; KO, knockout; MACS, magnetic-activated cell sorting; MFI, mean fluorescent intensity; mRNA, messenger RNA; PspA, pneumococcal surface protein A; TSLP, thymic stromal lymphopoietin; WT, wild type.

Although IgA Ab-forming cells were significantly decreased in spleen of TSLPR-KO mice (Figure 3b), the IgA-producing ability of DCs in spleen did not seem to be affected by the absence of TSLP-TSLPR signaling (Figure 5a,b). A previous study ${ }^{33}$ clearly showed that after intranasal immunization, high-affinity IgA memory cells generated in NALT migrate into non-mucosal lymphoid tissues such as spleen via the systemic circulation. Thus, we speculate that, following nasal 


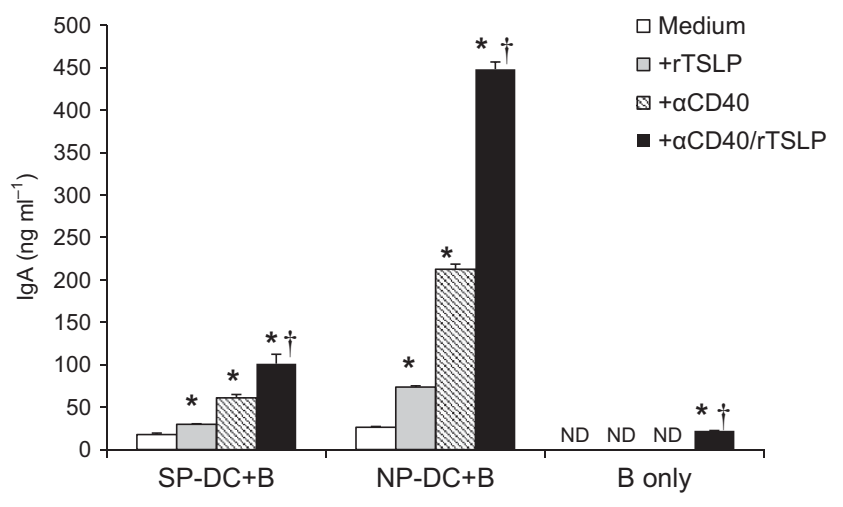

Figure 6 TSLP enhances IgA production in a DC-B-cell co-culture system. Purified WT splenic IgD ${ }^{+}$B cells were co-cultured with or without autologous WT splenic or nasal CD11 ${ }^{+}$DCs in a ratio of 2:1. Cells were cultured in the presence or absence of rTSLP $\left(20 \mathrm{ng} \mathrm{ml}^{-1}\right)$ and anti-CD40 $\mathrm{mAb}\left(\alpha \mathrm{CD} 40 ; 2.5 \mu \mathrm{g} \mathrm{ml}^{-1}\right)$. After 7 days, the concentrations of IgA in the culture supernatants were determined by ELISA. Data are expressed as means \pm s.e.m. ( $n=3$ per group). ${ }^{\star} P<0.05$ vs. medium only; ${ }^{\dagger} P<0.05$ vs. $+\alpha C D 40$. Data are representative of three independent experiments. DC, dendritic cells; ELISA, enzyme-linked immunosorbent assay; mAb, monoclonal $\mathrm{Ab}$; ND, not detected; TSLP, thymic stromal lymphopoietin; WT, wild type.

immunization with PspA plus CT, Ag-specific IgA-producing cells are primarily generated in nasal mucosal tissues with the help of nearby mucosal DCs, after which they further migrate into the general circulation and then to spleen.

In contrast to the PspA-specific IgA responses described above, PspA-specific systemic IgG responses were similar between WT mice and those immunized with PspA plus CT in the absence of TSLP signaling. This result suggests that mucosal DCs are primarily responsible for mucosal IgA responses, not systemic IgG responses, when Ag plus a Th2-type adjuvant such as CT is delivered by the nasal route. Indeed, several lines of evidence indicate that specific subsets of DCs, such as $\mathrm{CCR}^{+}{ }^{+} \mathrm{CCR}^{+}{ }^{+}$DCs in nasal or TLR $5{ }^{+}$DCs in intestinal lamina propria mucosa, are involved in IgA induction after nasal immunization or bacterial stimulation. ${ }^{34,35}$ We consider it possible that non-dendritic immune cells have a role in eliciting IgG responses after nasal immunization. Collectively, our findings clearly support the need for a mucosal DCtargeting immunization strategy to induce Ag-specific IgA responses.

The precise mechanisms by which nasal immunization with PspA plus CT enhances TSLP expression in respiratory tissues and DCs remain unclear. Previous studies suggest that expression of TSLP is regulated by nuclear factor- $\kappa \mathrm{B}$ and can be induced by exposure to viral, bacterial, parasitic products, inflammatory cytokines, or Th2 cell-associated cytokines such as IL-4 and IL-13. ${ }^{6,36,37}$ Here, TSLP expression in respiratory tissue was enhanced by nasal immunization with PspA plus CT, but not with PspA only, CT only, or PspA plus a Th1-type adjuvant (CpG ODN). Therefore, we speculate that the cytokine signaling cascade triggered by the bacterial Ag plus a Th2-type adjuvant rather than stimulation by the Ag itself induces TSLP expression. Supporting this notion, we found that nasal PspA-cCHP (nanogel), which produces Th2-type immune responses in mice and macaques, ${ }^{28,38}$ also upregulated TSLP expression in respiratory tissue (data not shown). However, whether this response is dependent on the nature of $\mathrm{Ag}$ or adjuvant needs to be further investigated.

Here, we demonstrated that IL-6 has an important role in TSLP-mediated IgA enhancement in in vitro DC-B cell coculture experiments. Compared with B cells alone, DCs plus B cells showed enhanced TSLP-mediated stimulation of IgA and IL-6 production in culture supernatants; this effect was more marked for NP-DCs than for SP-DCs (Figures 6 and 7). The TSLP-mediated stimulation of IgA production was largely diminished after the addition of IL-6-neutralizing $\mathrm{Ab}$ to the DC-B cell co-culture medium, suggesting that TSLP induction of IgA is IL-6-dependent. IL-6 is reported to promote IgA production from isotype-committed $\mathrm{B}$ cells $\mathrm{s}^{39}$ and is important for the development of mucosal $\mathrm{Ab}$ responses in vivo. ${ }^{40}$ It is therefore plausible that TSLP-stimulated DCs led to IL-6 production and subsequently promoted the differentiation of $\mathrm{IgA}^{+} \mathrm{B}$ cells into IgA-producing cells. In addition, both IgA and IL-6 production were moderately enhanced when WT naive B cells only were cultured with anti-CD40 and rTSLP, suggesting that not only DCs, but also B cells, are involved in the IgA-enhancing ability of TSLP. Further investigation is needed to identify the role of TSLP-TSLPR signaling in IgA production by B cells; additional factors produced by DCs or B cells might support TSLP-mediated IgA production. In other in vitro experiments, we found that IL-6 is a highly TSLP-dependent gene and that BAFF, iNOS, and IL-10 are directly regulated by the TSLP-TSLPR pathway (data not shown). How these molecules are regulated by TSLP-TSLPR signaling in vivo and further details of the underlying molecular mechanisms will be studied in the near future.

To summarize, here we have elucidated the critical role of TSLP-TSLPR signaling in Ag-specific IgA responses after nasal immunization with PspA plus CT in mice. Our findings suggest that TSLP production by respiratory epithelial cells and mucosal DCs increases in response to PspA plus CT nasal immunization and that this stimulates DC activation and maturation in mucosal tissues, where TSLP-responsive mucosal DCs enhance Ag-specific IgA production. By using in vitro DC-B cell co-cultures, we found that TSLP-mediated induction of IL-6 is important for enhancing IgA production from DC cells. Together our results suggest that future mucosal vaccines and adjuvants against pathogens should activate and target TSLP-TSLPR signaling in the mucosal compartments to induce more effective Ag-specific IgA responses.

\section{METHODS}

Mice. BALB/c mice (age, 6-7 weeks) were purchased from CLEA Japan (Tokyo, Japan) and TSLPR-KO mice were established by Dr Steven F. Ziegler (Benaroya Institute, USA) and kindly provided by Dr Nakae Susumu (The Institute of Medical Science, The University of Tokyo). All experiments were conducted in accordance with the guidelines provided by the Animal Care and Use Committees of The University of Tokyo and were approved by the Animal Committee of The Institute of Medical Science of The University of Tokyo. 

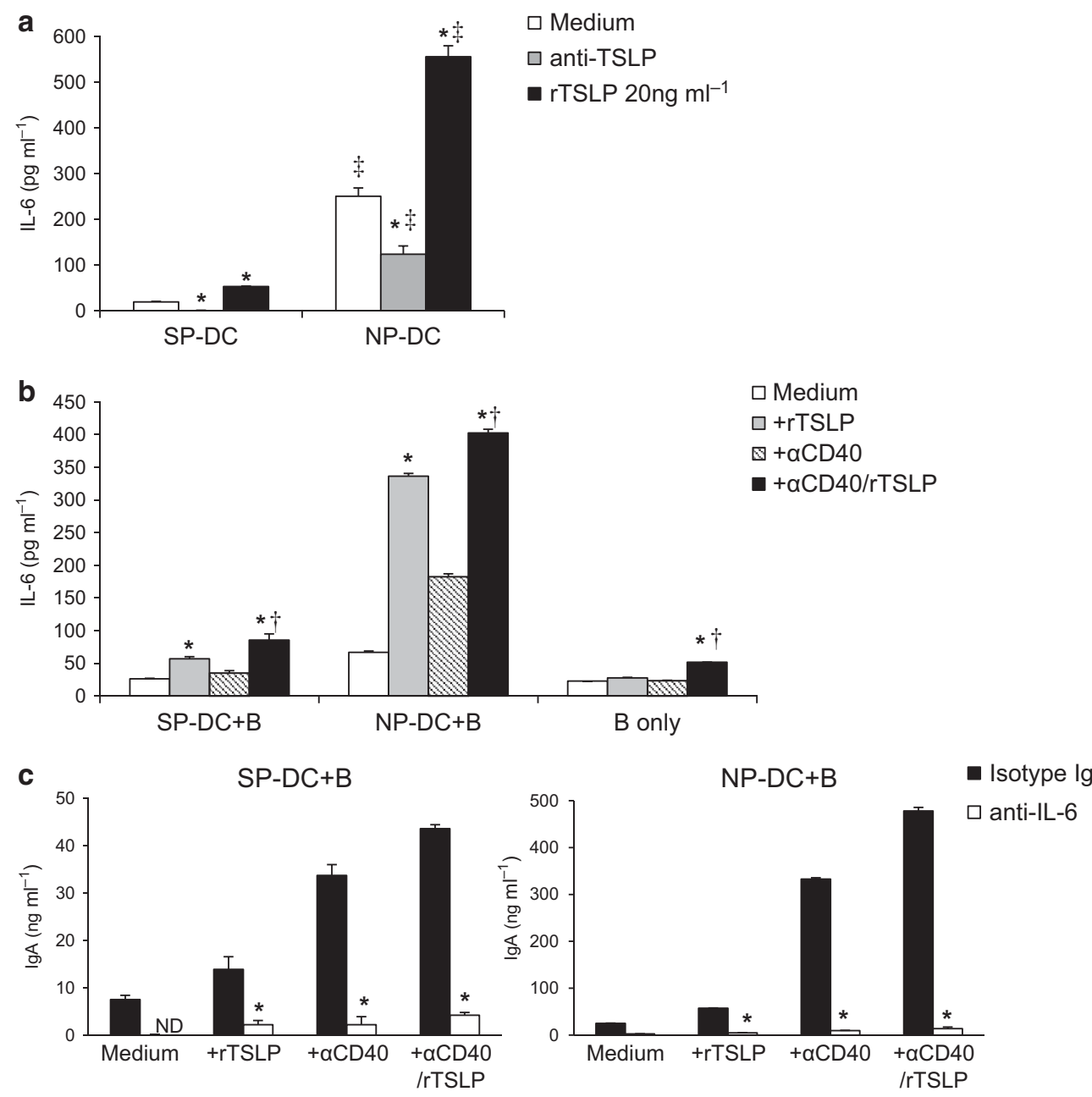

Figure 7 Role of IL-6 in IgA-enhancing ability of TSLP. (a) Purified splenic or nasal CD11C ${ }^{+}$DCs were cultured in the presence of stimulators (antiTSLP; $1 \mu \mathrm{g} \mathrm{ml}^{-1}$, rTSLP; $20 \mathrm{ng} \mathrm{ml}^{-1}$ ). (b) Purified WT splenic lgD ${ }^{+}$B cells were co-cultured with or without autologous WT splenic or nasal CD11 ${ }^{+}$DCs at $^{-1}$ a ratio of $2: 1$. Cells were cultured in the presence or absence of $\operatorname{rTSLP}\left(20 \mathrm{ng} \mathrm{m}^{-1}\right)$ and anti-CD $40 \mathrm{mAb}^{2}\left(2.5 \mu \mathrm{g} \mathrm{ml}^{-1}\right)$. (c) Purified naive WT splenic lgD ${ }^{+}$ B cells and splenic or nasal CD11 $\mathrm{c}^{+}$DCs were co-cultured with (white) or without (black) anti-IL-6 mAb (10 $\left.\mu \mathrm{g} \mathrm{ml}^{-1}\right)$ in the presence or absence of $\mathrm{rTSLP}$ and anti-CD $40 \mathrm{mAb}$. At the end of $48 \mathrm{~h}(\mathbf{a})$ or 7 days $(\mathbf{b}, \mathbf{c})$ of culture, supernatants were collected and IL- $6(\mathbf{a}, \mathbf{b})$ and IgA (c) concentrations were determined by ELISA. Data are expressed as means \pm s.e.m. ( $n=3$ per group). ${ }^{\star} P<0.05$ vs. (a,b) medium only, (c) isotype Ig control; ${ }^{\dagger} P<0.05$ vs. $+\alpha C D 40 ;{ }^{\ddagger} P<0.05$ vs. SP-DC. Data are representative of two $(\mathbf{a})$ or three $(\mathbf{b}, \mathbf{c})$ independent experiments. DC, dendritic cells; ELISA, enzyme-linked immunosorbent assay; IL, interleukin; mAb, monoclonal Ab; ND, not detected; rTSLP, recombinant TSLP; TSLP, thymic stromal lymphopoietin; WT, wild type.

Nasal immunization and sample collection. Recombinant PspA of $S$. pneumoniae Rx1, which belongs to PspA family 1 and clade 2, was prepared as described previously, ${ }^{38,41}$ and CT (List Biological Laboratory, Campbell, CA, USA) was used as a nasal adjuvant. Mice were nasally immunized with $5 \mu \mathrm{g}$ PspA and $1 \mu \mathrm{g}$ CT at weekly intervals for 3 consecutive weeks. For the control groups, mice were nasally administered with $5 \mu \mathrm{g}$ PspA or $1 \mu \mathrm{g}$ CT alone or $5 \mu \mathrm{g}$ PspA plus $10 \mu \mathrm{g}$ CpG ODN. Serum, nasal wash samples, and bronchoalveolar fluid were collected 1 week after the last immunization, as previously described. ${ }^{5,28}$ Nasal washes were collected by gently flushing the NP with $200 \mu \mathrm{l}$ of sterile PBS, and broncho-alveolar fluid were collected by gently flushing the lung with $1 \mathrm{ml}$ of sterile PBS.

RNA and protein quantification. Total RNA was prepared from mouse tissues or magnetic-activated cell sorting-isolated $\mathrm{CD} 11 \mathrm{c}^{+}$ DCs by using TRIzol regent (Invitrogen, Carlsbad, CA, USA), and then complimentary DNA was synthesized by using PrimeScript RT Master Mix (Takara, Shiga, Japan) according to the manufacturer's protocol. The complimentary DNA was analyzed by quantitative RT-PCR by using Fast SYBR Green Master Mix and the Step One Plus Real-Time PCR System (Applied Biosystems, Carlsbad, CA, USA). Expression levels were normalized to that of $\beta$-actin. The PCR primers used are listed in Supplementary Table S1.

The concentration of TSLP in broncho-alveolar fluid samples was measured by using a TSLP ELISA kit (R\&D Systems, Minneapolis, MN, USA).

The concentration of IL-6 in the culture supernatant of DC-B cell co-culture experiments was determined by using a mouse IL-6 Duoset ELISA kit (R\&D Systems), and the concentration of total IgA in the culture supernatant was measured by standard ELISA as previously reported. ${ }^{42}$ The detection limit level for IgA was $1.95 \mathrm{ng} \mathrm{ml}^{-1}$.

Cell preparation and flow cytometric analysis. Mononuclear cells from spleen, NALT, and cervical lymph node were isolated aseptically by mechanical dissociation through stainless steel screens. ${ }^{34}$ To exclude contamination from macrophages, lung tissues were vigorously washed by lavage with ice-cold PBS as previously described. ${ }^{43} \mathrm{NP}$ and lung tissues were excised, teased apart, and enzymatically digested 
with collagenase type IV (Sigma-Aldrich, St Louis, MO, USA), and then mononuclear cells were purified by discontinuous Percoll gradient centrifugation (Amersham Biosciences, Piscataway, NJ, USA). Isolated cells were stained with fluorescent Abs specific for CD11c, CD40, I-A ${ }^{\mathrm{d}}$, $\mathrm{CD} 3 \mathrm{e}$, or CD8 $\alpha$ (all from BD Biosciences, Oxford, UK), CD11b, CD86, CD19, CD127 (IL-7R $\alpha$ ), CD103, I-A/I-E (MHC II), or F4/80 (all from BioLegend, San Diego, CA, USA) or PE-anti-mouse TSLPR (R\&D Systems). Flow cytometric analysis was performed and analyzed by using the FACSCalibur and FACSCanto II systems (BD Biosciences).

PspA-specific ELISA. Ninety-six-well plates were coated with $1 \mu \mathrm{g} \mathrm{ml}^{-1} \mathrm{PspA}$ in PBS overnight at $4{ }^{\circ} \mathrm{C}$. After blocking of the plate with $1 \%$ BSA in PBS-Tween 20, two-fold serial dilutions of samples were added and incubated for $2 \mathrm{~h}$ at room temperature (RT). After washing of the samples, horseradish peroxidase-conjugated goat antimouse IgG, IgA, IgG1, or IgG2a (SouthernBiotech, Birmingham, AL, USA) was added and incubated for $2 \mathrm{~h}$ at RT. The reaction was developed by using the TMB Microwell Peroxidase Substrate System (XPL, Gaithersburg, MD, USA). End-point titers were expressed as the reciprocal $\log 2$ of the last dilution that gave an OD450 of 0.1 greater than the negative control.

PspA-specific ELISPOT. Mononuclear cells from relevant tissues were isolated as described above and prepared cells were subjected to an ELISPOT assay to determine the numbers of PspA-specific Ab-forming cells. Cells were processed on 96-well filtration plates (Millipore, Billerica, MA) coated with $1 \mu \mathrm{g} \mathrm{ml}^{-1}$ PspA. The numbers of PspA-specific Ab-forming cells were quantified using ZEISS KS ELISPOT software (Oberkochen, Germany).

Bacterial strain and infection. We used the kanamycin-resistant pneumococcal strain S. pneumoniae Xen10 (Caliper Life Sciences, Waltham, MA, USA) derived from the WT strain A66.1, which expresses PspA of family 1, clades 1, and 2. The virulence of S. pneumoniae Xen 10 is comparable to that of the parent strain. ${ }^{44}$ The S. pneumoniae strains were grown in Brain Heart Infusion (BHI) broth (BD Healthcare, Franklin Lakes, NJ) at $37^{\circ} \mathrm{C}$ in $5 \% \mathrm{CO} 2$. Mice were challenged with $S$. pneumoniae strain Xen10 1 week after the last immunization. Sublethal $\left(5 \times 10^{4} \mathrm{CFU}\right)$ challenge doses diluted in $40 \mu \mathrm{l}$ sterile PBS were administered nasally to isoflurane-anesthetized mice. Three days after challenge, NP and lung tissues were harvested and washed in $1 \mathrm{ml}$ sterile PBS. The numbers of bacterial colonies were determined by plating samples on Tryptic Soy Agar plates (BD Healthcare) containing kanamycin $\left(200 \mu \mathrm{g} \mathrm{ml}^{-1}\right)$ and incubation at $37^{\circ} \mathrm{C}$ overnight.

DC and B-cell isolation. For DC isolation, mononuclear cells were isolated from spleen, cervical lymph node, NALT, NP, and lung tissues as described above, and CD11c-positive cells were enriched by using anti-CD11c-labeled MicroBeads (Miltenyi Biotec, Auburn, CA, USA) according to the manufacturer's instructions. The purity of positively selected cells was $\sim 85 \%$. Isolated $\mathrm{CD}_{11} \mathrm{c}^{+}$DCs were subsequently subjected to mRNA analysis or in vitro DC-B cell co-culture. CD11cpositive cells enriched by magnetic-activated cell sorting, the proportion of macrophages $\left(\mathrm{CD} 11 \mathrm{c}^{\text {int }} \mathrm{MHC} \mathrm{II}{ }^{+} \mathrm{CD} 11 \mathrm{~b}^{+} \mathrm{F} 4 / 80^{+}\right.$) ranged from $2-7 \%$ (spleen; $2.1 \pm 0.4 \%$, NALT; $1.9 \pm 1.1 \%$; NP; $7.1 \pm 2.6 \%$, lung; $3.6 \pm 1.3 \%$ ), and the majority of cells were conventional DCs (CD11 $\mathrm{c}^{\text {high }} \mathrm{MHC} \mathrm{II}^{+} \mathrm{F} 4 / 80^{-}$).

Naive B cells were isolated from the spleen of naive WT mice by incubation of cell suspensions with biotin-anti-mouse $\operatorname{IgD}^{\mathrm{a}}$ (BD Biosciences), and then $\mathrm{IgD}^{+}$naive $\mathrm{B}$ cells were positively selected by using anti-biotin-coated Microbeads (Miltenyi Biotec). The purity of naive B-cell preparations was $>95 \%$.

Stimulation of DCs in vitro. Magnetic-activated cell sorting-purified splenic or nasal CD11c ${ }^{+}$DCs $\left(5 \times 10^{4}\right.$ per well $)$ were cultured with anti-mouse TSLP $\left(1 \mu \mathrm{g} \mathrm{ml}^{-1}\right.$; clone $152614, \mathrm{R} \& \mathrm{D}$ Systems $)$ or recombinant TSLP (20 $\mathrm{ng} \mathrm{ml}^{-1}$; R\&D Systems) in triplicate wells.
Culture supernatants were harvested at $48 \mathrm{~h}$, and IL- 6 levels were measured by ELISA.

In vitro DC-B cell co-cultures. $\mathrm{CD} 11 \mathrm{c}^{+} \mathrm{DCs}$ were pooled from spleen or NP tissue of five or six mice and were co-cultured with autologous naive $\mathrm{IgD}^{+} \mathrm{B}$ cells from spleen at a ratio of 1:2 in triplicate wells of round bottom 96-well plates. Cells were cultured in the presence or absence of $2.5 \mu \mathrm{g} \mathrm{ml}^{-1}$ anti-CD40 monoclonal Ab (clone 3/23, BD Biosciences) and recombinant TSLP (R\&D Systems) for 7 days at $37^{\circ} \mathrm{C}$ and concentrations of total IgA and IL- 6 in the culture supernatants were measured by ELISA.

For the neutralization of IL- $6,10 \mu \mathrm{g} \mathrm{ml}^{-1}$ of rat $\operatorname{IgG} 1 \kappa$ isotype control (clone R3-34; BD Biosciences) or anti-IL-6 monoclonal Ab (clone MP5-20F3; BD Biosciences) was added to the medium of the DC-B cell co-cultures, and the concentrations of IgA and IL-6 in the supernatants were measured 7 days later.

RPMI-1640 (Nakalai Tesque, Kyoto, Japan) supplemented with $10 \%$ heat inactivated fetal bovine serum (Gibco, Carlsbad, CA, USA), $50 \mathrm{U} \mathrm{ml}^{-1}$ penicillin plus $50 \mu \mathrm{g} \mathrm{ml}^{-1}$ streptomycin (Gibco), $55 \mu \mathrm{M}$ 2-mercaptoethanol (Gibco), and $1 \mathrm{~mm}$ sodium pyruvate (Gibco) were used in cell cultures as complete medium.

Statistical analysis. The results are presented as the means \pm 1 s.d. or s.e.m. Two-tailed Student's $t$-test was used for comparisons between the groups. $P$ values $<0.05$ were considered significant.

SUPPLEMENTARY MATERIAL is linked to the online version of the paper at http://www.nature.com/mi

\section{ACKNOWLEDGMENTS}

We are grateful to Professor Susumu Nakae (The University of Tokyo) for providing TSLPR-KO mice. This work was supported by grants from the Health and Labour Sciences Research Grant Adjuvant Database Project from the Japanese Ministry of Health, Labour, and Welfare (H.K.); the Core Research for Evolutional Science and Technology Program of the Japan Science and Technology Agency (H.K.); the Ministry of Education, Culture, Sports, Science, and Technology of Japan Grant-in-Aid for Scientific Research S 23229004] (H.K.) and Research Activity Start-up [26893047] (Y.K.); the Young Researcher Overseas Visits Program for Vitalizing Brain Circulation of the Japan Society for the Promotion of Science (H.K.); and the MEXT Translational Research Network Program (at the University of Tokyo) Seeds B (H.K.).

\section{DISCLOSURE}

The authors declared no conflict of interest

c 2017 Society for Mucosal Immunology

\section{REFERENCES}

1. Fujkuyama, Y. et al. Novel vaccine development strategies for inducing mucosal immunity. Expert Rev. Vaccines 11, 367-379 (2012).

2. Kiyono, H. \& Fukuyama, S. NALT- versus Peyer's-patch-mediated mucosal immunity. Nat. Rev. Immunol. 4, 699-710 (2004).

3. Cerutti, A. The regulation of IgA class switching. Nat. Rev. Immunol. 8, 421-434 (2008)

4. Strugnell, R.A. \& Wijburg, O.L. The role of secretory antibodies in infection immunity. Nat. Rev. Microbiol. 8, 656-667 (2010).

5. Fukuyama, Y. et al. Secretory-lgA antibodies play an important role in the immunity to Streptococcus pneumoniae. J. Immunol. 185, 1755-1762 (2010).

6. Ziegler, S.F. \& Artis, D. Sensing the outside world: TSLP regulates barrier immunity. Nat. Immunol. 11, 289-293 (2010).

7. Kashyap, M., Rochman, Y., Spolski, R., Samsel, L. \& Leonard, W.J. Thymic stromal lymphopoietin is produced by dendritic cells. J. Immunol. 187, 1207-1211 (2011).

8. Liu, Y.J. et al. TSLP: an epithelial cell cytokine that regulates T cell differentiation by conditioning dendritic cell maturation. Annu. Rev. Immunol. 25, 193-219 (2007). 
9. Reche, P.A. et al. Human thymic stromal lymphopoietin preferentially stimulates myeloid cells. J. Immunol. 167, 336-343 (2001).

10. Ziegler, S.F. \& Liu, Y.J. Thymic stromal lymphopoietin in normal and pathogenic T cell development and function. Nat. Immunol. 7, 709-714 (2006).

11. Al-Shami, A., Spolski, R., Kelly, J., Keane-Myers, A. \& Leonard, W.J. A role for TSLP in the development of inflammation in an asthma model. J. Exp. Med. 202, 829-839 (2005).

12. Ziegler, S.F. Thymic stromal lymphopoietin and allergic disease. J. Allergy Clin. Immunol. 130, 845-852 (2012).

13. Ito, T. et al. TSLP-activated dendritic cells induce an inflammatory Thelper type 2 cell response through OX40 ligand. J. Exp. Med. 202, 1213-1223 (2005).

14. Zhou, B. et al. Thymic stromal lymphopoietin as a key initiator of allergic airway inflammation in mice. Nat. Immunol. 6, 1047-1053 (2005).

15. He, B. et al. Intestinal bacteria trigger T cell-independent immunoglobulin $\mathrm{A}(2)$ class switching by inducing epithelial-cell secretion of the cytokine APRIL. Immunity 26, 812-826 (2007).

16. Xu, W. et al. Epithelial cells trigger frontline immunoglobulin class switching through a pathway regulated by the inhibitor SLPI. Nat. Immunol. 8, 294-303 (2007).

17. Sugimoto, H. et al. Thymic stromal lymphopoietin plays an adjuvant role in BCG-mediated CD8 $(+)$ cytotoxic T cell responses through dendritic cell activation. Clin. Immunol. 136, 205-216 (2010).

18. Van Roey, G.A., Arias, M.A., Tregoning, J.S., Rowe, G. \& Shattock, R.J. Thymic stromal lymphopoietin (TSLP) acts as a potent mucosal adjuvant for HIV-1 gp140 vaccination in mice. Eur. J. Immunol. 42, 353-363 (2012).

19. Briles, D.E. et al. Immunizations with pneumococcal surface protein $A$ and pneumolysin are protective against pneumonia in a murine model of pulmonary infection with Streptococcus pneumoniae. J. Infect. Dis. 188, 339-348 (2003).

20. Grevers, G. Challenges in reducing the burden of otitis media disease: an ENT perspective on improving management and prospects for prevention. Int. J. Pediatr. Otorhinolaryngol. 74, 572-577 (2010).

21. Crain, M.J. et al. Pneumococcal surface protein A (PspA) is serologically highly variable and is expressed by all clinically important capsular serotypes of Streptococcus pneumoniae. Infect. Immun. 58, 3293-3299 (1990).

22. Yamamoto, M. et al. A nontoxic adjuvant for mucosal immunity to pneumococcal surface protein A. J. Immunol. 161, 4115-4121 (1998).

23. Wu, H.Y., Nahm, M.H., Guo, Y., Russell, M.W. \& Briles, D.E. Intranasal immunization of mice with PspA (pneumococcal surface protein A) can prevent intranasal carriage, pulmonary infection, and sepsis with Streptococcus pneumoniae. J. Infect. Dis. 175, 839-846 (1997).

24. Klinman, D.M., Barnhart, K.M. \& Conover, J. CpG motifs as immune adjuvants. Vaccine 17, 19-25 (1999).

25. Kitajima, M. \& Ziegler, S.F. Cutting edge: identification of the thymic stromal lymphopoietin-responsive dendritic cell subset critical for initiation of type 2 contact hypersensitivity. J. Immunol. 191, 4903-4907 (2013).

26. Bell, B.D. et al. The transcription factor STAT5 is critical in dendritic cells for the development of TH2 but not TH1 responses. Nat. Immunol. 14, 364-371 (2013).
27. Mosconi, I. et al. Intestinal bacteria induce TSLP to promote mutualistic T-cell responses. Mucosal Immunol. 6, 1157-1167 (2013).

28. Kong, I.G. et al. Nanogel-based PspA intranasal vaccine prevents invasive disease and nasal colonization by Streptococcus pneumoniae. Infect. Immun. 81, 1625-1634 (2013).

29. Melum, G.R. etal. A thymic stromal lymphopoietin-responsive dendritic cell subset mediates allergic responses in the upper airway mucosa. J. Allergy Clin. Immunol. 134, 613-621 e617 (2014).

30. Massacand, J.C. et al. Intestinal bacteria condition dendritic cells to promote IgA production. PLoS One 3, e2588 (2008).

31. Iwasaki, A. Mucosal dendritic cells. Annu. Rev. Immunol. 25, 381-418 (2007).

32. Spadoni, I., lliev, I.D., Rossi, G. \& Rescigno, M. Dendritic cells produce TSLP that limits the differentiation of Th17 cells, fosters Treg development, and protects against colitis. Mucosal Immunol. 5, 184-193 (2012).

33. Shimoda, M. et al. Isotype-specific selection of high affinity memory B cells in nasal-associated lymphoid tissue. J. Exp. Med. 194, 1597-1607 (2001).

34. Fukuyama, Y. et al. Potential roles of CCR5 $(+)$ CCR6 $(+)$ dendritic cells induced by nasal ovalbumin plus Flt3 ligand expressing adenovirus for mucosal IgA responses. PLoS One 8, e60453 (2013).

35. Uematsu, S. et al. Regulation of humoral and cellular gut immunity by lamina propria dendritic cells expressing toll-like receptor 5. Nat. Immunol. 9, 769-776 (2008).

36. Lee, H.C. \& Ziegler, S.F. Inducible expression of the proallergic cytokine thymic stromal lymphopoietin in airway epithelial cells is controlled by NFkappaB. Proc. Natl Acad. Sci. USA 104, 914-919 (2007).

37. Allakhverdi, Z. et al. Thymic stromal lymphopoietin is released by human epithelial cells in response to microbes, trauma, or inflammation and potently activates mast cells. J. Exp. Med. 204, 253-258 (2007).

38. Fukuyama, Y. et al. Nanogel-based pneumococcal surface protein A nasal vaccine induces microRNA-associated Th17 cell responses with neutralizing antibodies against Streptococcus pneumoniae in macaques. Mucosal Immunol. 8, 1144-1153 (2015).

39. Beagley, K.W. et al. Interleukins and IgA synthesis. Human and murine interleukin 6 induce high rate IgA secretion in IgA-committed B cells. J. Exp. Med. 169, 2133-2148 (1989).

40. Ramsay, A.J. et al. The role of interleukin-6 in mucosal IgA antibody responses in vivo. Science 264, 561-563 (1994).

41. Briles, D.E. et al. Intranasal immunization of mice with a mixture of the pneumococcal proteins PsaA and PspA is highly protective against nasopharyngeal carriage of Streptococcus pneumoniae. Infect. Immun. 68, 796-800 (2000).

42. Kotani, Y. et al. Role of Lactobacillus pentosus Strain b240 and the toll-like receptor 2 axis in Peyer's patch dendritic cell-mediated immunoglobulin A enhancement. PLoS One 9, e91857 (2014).

43. Naito, T. et al. Lung dendritic cells have a potent capability to induce production of immunoglobulin A. Am. J. Respir. Cell Mol. Biol. 38, 161-167 (2008).

44. Darrieux, M. et al. Fusion proteins containing family 1 and family 2 PspA fragments elicit protection against Streptococcus pneumoniae that correlates with antibody-mediated enhancement of complement deposition. Infect. Immun. 75, 5930-5938 (2007). 